\title{
Fault disturbances classification analysis using adaptive neuro-fuzzy inferences system
}

\author{
Shahrani Shahbudin ${ }^{1}$, Murizah Kassim², Roslina Mohamad ${ }^{3}$, Saiful Izwan Suliman ${ }^{4}$, \\ Yuslinda Wati Mohamad Yusof ${ }^{5}$ \\ Faculty of Electrical Engineering, Universiti Teknologi MARA, Malaysia
}

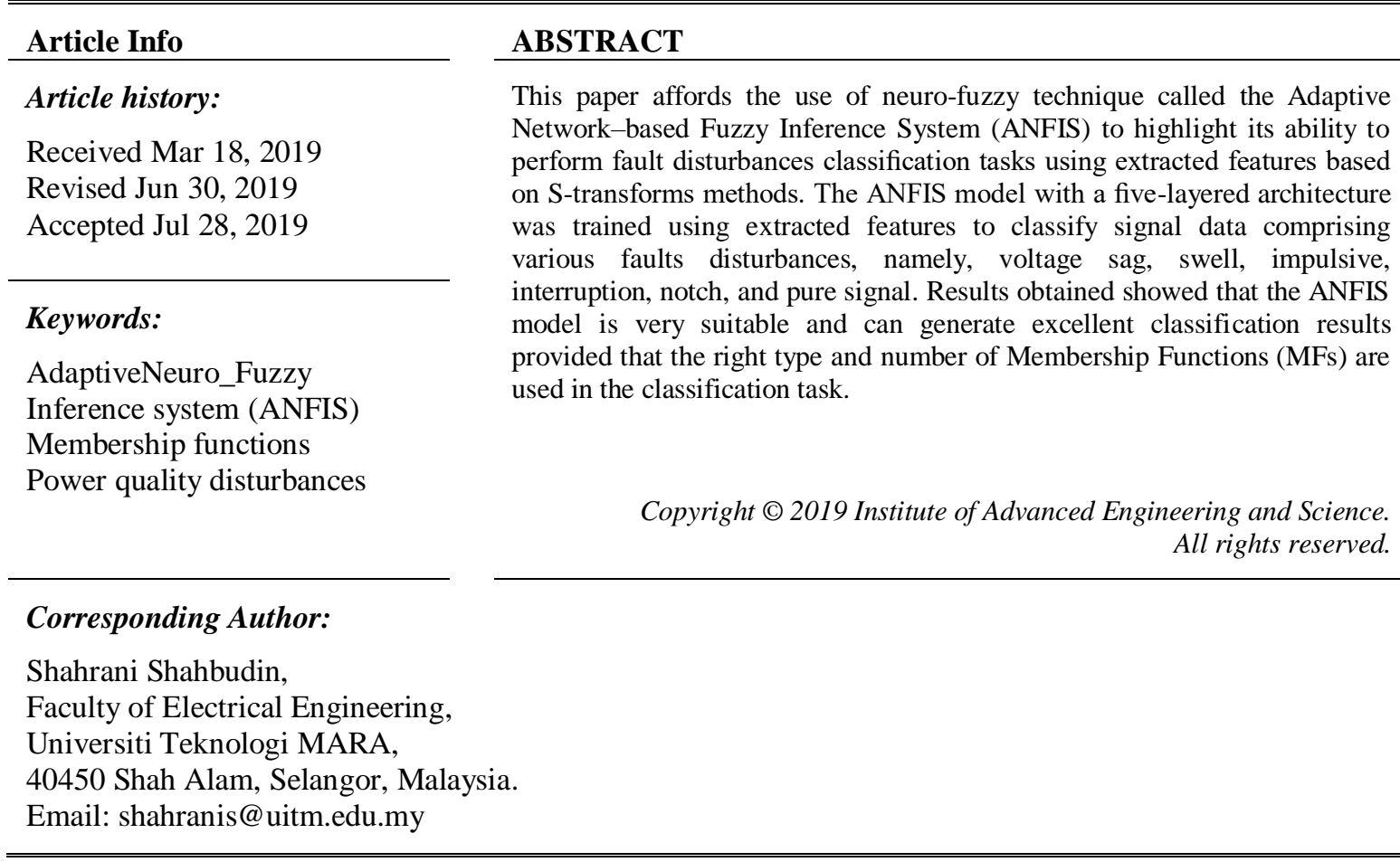

\section{INTRODUCTION}

In this era, the major concern in power system are to discover the power quality due to the impact of PQ disturbances (PQDs). The monitoring and analysis of PQD are generally performed with specialized measuring equipment based on the standards. Based on the research [1, 3, 5], most of the PQDs where generalized in wavelet transform before extracting to the next stage. During the generalized, the minimum frequency is fixed in separation threshold in prior and generates the substantial redundant information.

Moreover, most of the research paper $[2,3,6]$, for classify the PQDs it use support vector machine (SVM) after the extraction process of the data because the SVM are efficient in large classification problems and better in generalization properties compared to conventional classifiers but in others [1, 4, 5], where using the classifiers of data which are, decision tree, perception tree and also, random forest that consist a group of tree that used. It known as considering the standards and practical signal characteristics. Other than that, there are method proposed which contains the feature extraction (FE) and decision-making [7]. In FE, histogram are utilize and combine with a Discrete Wavelet Transform (DWT) that can detect the PQ while in the stage of decision, Extreme Learning Machine (ELM) classify the dataset of PQ which resultant of the performance detected is high [7].

The Adaptive Network-based Fuzzy Inference System (ANFIS) introduced by Jang [13, 14] is a well-known neural fuzzy controller with fuzzy inference capability had been implemented in various works [8-12]. ANFIS is based on fuzzy logic modeling and use ANN as the learning algorithm.

The aim of this work is to highlight the application of the ANFIS to perform fault disturbances classification using three set feature vectors extracted from the S-transform and to find the right type and 
number of Membership Functions (MF's) for used in ANFIS. This is to provide a fast action to supply power to the consumer if there is some disturbances occur in the power system.

The remaining parts are organized as follows. Detailed methodology is discussed in Section 2 Section 3 describes the results obtained. Finally, conclusions are given in Section 4.

\section{RESEARCH METHOD}

The flow chart of the proposed method is shown in Figure 1. In this study, three feature vectors extracted from the S-transform introduced in [15] were applied as inputs and classification stage with ANFIS implementation will be carried out using MATLAB software.

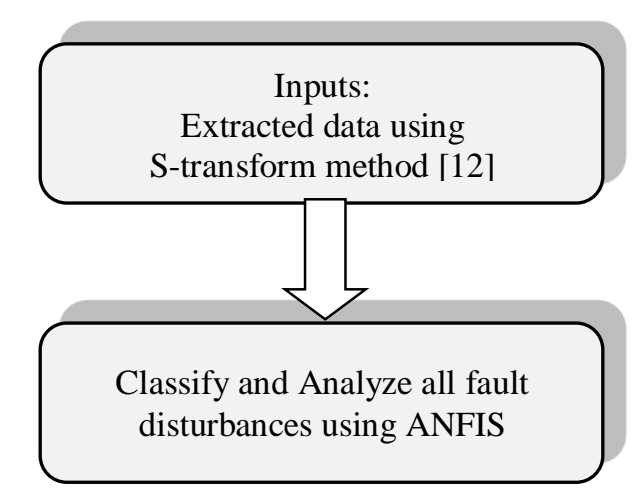

Figure 1. The flow of the proposed method

\subsection{S-transform Feature Extraction}

S-transform is one of the feature extraction methods based on time-frequency analysis. It extracts the pre-processing data into the most salient features that represent the power quality phenomenon [16]. The original formula for s-transform is shown in (1).

$$
S(\tau, f)=\frac{|f|}{\sqrt{2 \pi}} \int_{-\infty}^{\infty} x(t) e^{-\frac{(\tau-t)^{2} f^{2}}{2}} e^{-i 2 \pi f t} d t
$$

where: $f$ is frequency, $t$ and $\tau$ is time

The output from S-transform is a $m \times n$ matrix known as $\mathrm{S}$ matrix with column is referred to time and row is referred to frequency. Every element from this matrix is a complex value. Each row indicates the magnitude of S-transform with all the frequency at the same time and each column indicates the magnitude of S-transform from same frequency.

According to [15], the first step in implementing the feature extraction is to map the distorted data signal to S-transform domain. For non-stationary signal interference and noise, using the analysis of Stransform, feature vectors extracted from signal interference in the form of time-frequency representation of the curves.

A statistical technique is used to the amplitude of contour matrix of S-transform by using maximum amplitude and frequency amplitude plot. The resulting features are formulated as follows.

Amplitude factor, F1 as the first feature with the given by (2),

$$
F_{1}=1+s t d 1+s t d 2-\text { norm } 1-\text { norm } 2
$$

Where:

std1: Maximum value of standard deviation distortion signal

std2: Minimum value of standard deviation distortion signal

norm1: Maximum value of normal signal

norm2: Minimum value of normal signal

Second feature is given by the equation of,

$$
F 2=s t d 1-\text { norm } 1
$$


And the third feature is given by equation,

$$
F 3=\text { mean }\left(\text { mean }\left(a b s(d s)^{2}\right)\right)
$$

Where:

ds: absolute value of S-transform distortion signal

In this study, based on these formulas, three feature vectors extracted were used as inputs in which parameter F1 was assigned as input one, input two was represented by parameter F2, and parameter F3 represented input three. Table 1 depicts the sample of three inputs.

Table 1. Sample of The Input Data Extracted From S-Transform Method

\begin{tabular}{llrl} 
Fypes of & & & \\
Faults & F1 & F2 & F3 \\
\hline Sag & & & \\
Swell & 1.0573 & 0.0302 & 0.0009 \\
Impulsive & 1.0814 & 0.045 & 0.0018 \\
Interruption & 1.0936 & 0.059 & 0.0013 \\
Notch & 1.0449 & 0.0386 & 0.0009 \\
Pure & 1.0703 & 0.0355 & 0.0013 \\
\hline
\end{tabular}

\subsection{Adaptive Neuro-Fuzzy Inference System}

ANFIS technique has been considered in this work because it had been stated as one of the simplest and has a well-defined mathematical model technique to determine the classification of fault [17-19]. It is a hybrid combination of Adaptive Neural Network (ANN) and Fuzzy Inference System (FIS) [12]. FIS use a membership mapping modeling of input to determine its output by a membership function parameters. This membership function parameter can be adjusted to get the targeted values. ANN function as a learning algorithm to the system which gives fast convergence and more accurate in ANFIS target. ANN is adapted so that it can computes the membership function parameters that best allow the associated FIS to track the given input/output data since the FIS only applied to only modeling systems whose rule structure is essentially predetermined by the user's interpretation [12].

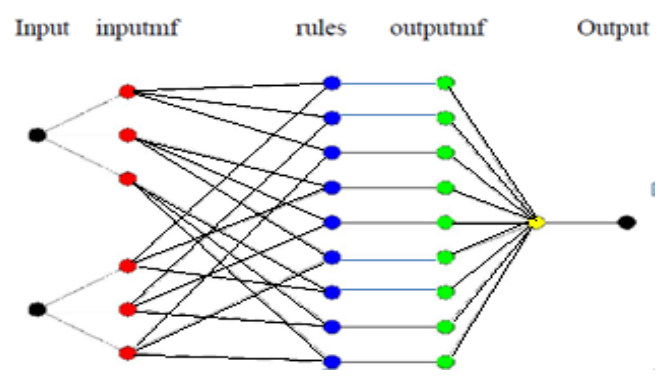

Figure 2. Architecture of ANFIS

addition, ANFIS is applied in this work because it give a high precision, easy, and effective technique $[12,13]$.

The architecture of ANFIS is depicted in Figure 2 [20]. It consists of five layers with two inputs and one output based on the Takagi-Sugeno model.

\section{Layer 1: Fuzzification}

This layer is located at the first nodes at the receiving of input data or signal. It adjusted the input signal and performed a Membership Function (MF) in (5). The MF parameters are adjusted according to the user interpretation.

$$
\alpha_{i}=k_{a}(x)
$$

where $\alpha_{\mathrm{i}}$ is membership function of $k_{a}(x)$ and a is linguistic label associated with the label. 


\section{Layer 2: IF-THEN Stage Rule}

In this stage, the signal that has through a fuzzification from the first layer will represent as the firing strength of the IF-THEN rule. The output is multiple of inputs and it performs the fuzzy AND rules.

$$
W_{i}=R_{a i}(x)^{*} R_{b i}(y) \quad i=1,2,3, \ldots
$$

\section{Layer 3: Normalization}

The input for normalization layer is the output from the IF-THEN rule layer. In this layer, the normalized firing strength will be calculated. Every gained signal is divided by the total of gained signal using below equation.

$$
H_{i}=\frac{R i}{\sum_{i=1}^{n} R}
$$

\section{Layer 4: Defuzzification}

In this layer, parameters of output are adjusted because of each node is an adaptive node. Normalized signal is gained by the linear function of MF to the output signal.

$$
f_{i}=N_{i}\left(p_{i} x+q_{i} y+r_{i}\right) \quad i=1,2,3 \ldots
$$

$\mathrm{p}_{\mathrm{i}} \mathrm{x}, \mathrm{q}_{\mathrm{i}} \mathrm{y}$ and $\mathrm{r}_{\mathrm{i}}$ are the MF parameters for the linear signal.

\section{Layer 5: Neuron Addition}

This is the last layer for ANFIS. In this layer it will calculate the entire defuzzification signal since it has only one node, with the summation of the input signal.

$$
O T=\sum_{i=1}^{n} f I
$$

\section{RESULTS AND ANALYSIS}

Hybrid learning algorithm is employed to determine the optimum values of the FIS parameters of the Sugeno-type model. Hybrid-learning is the combination of least-squares method and back-propagation. In the ANFIS, the training data were used to train (or tune) a fuzzy model. In this work, a collection of 264 samples divided into two part; $70 \%$ for training and 30\% for testing data.

Before training process, several parameters need to be set up first [21]. The numbers of Membership Functions (MF's) are assigned to each input of the ANFIS and were set to two and three MF's. The number of rules produces for two numbers of MF's is 8-rules and for three numbers of MF's, rules generated is 27rules respectively. To select the best model fit based on the type of MF's, two membership functions has been selected which are: the Gaussian function (gaussmf) and generalized-bell function (gbellmf). The step size is the length of each gradient transition in the parameter space. In this project, the parameter of step size is set as follows: the initial step size value was set to 0.1 ; the step size-decreasing rate is set equal to 0.9 , and the step increase rate was 1.1. Epochs or number of iteration is set to 100. Meanwhile, the hybrid learning method was employed, and the linear output type is selected.

Feature saliency provides a mean for choosing the best features for classification [22]. Since the feature vectors used as input may have different levels of relevancy, the changes that happen after training the MF's are examined. Figure 3 shows the plots of the MF's before and after training. Referring to Figure 3, changes of the MF were noted for the case of two and three MF's using the "gbellmf' and "gaussmf". In overall, for all the cases, most of MF's for feature 3 were changed after training process. However, for the case of using three MF's with "gbellmf", MF's for feature 2 and feature 3 were changed, whereas no change was noted in other plots. These results imply that by implementing different numbers of MF's and choosing the right type of MF's, the classification results can be improved and in the case of the "gaussmf" it gave the best results when three MF's are in used.

In addition, the Root Mean Square Error (RMSE) for both training and testing were compared and tabulated in Table 2. RMSE error is the training and testing error for the analysis of the parameter. The error that produce from this analysis is taken to determine which parameter has the best (the least) error for the classification of the fault disturbances. It is obvious that by implementing different numbers and types of membership functions, the value of each error rate also changed. According to the Table 2, during training process, the ANFIS model with two MFs that used "gaussmf" had better training learning result compared to ANFIS with “gbellmf”. The smallest training error recorded was 0.2956. 
On the other hand, in term of testing process, in the case of the "gaussmf" it gave the best results when three MFs are in used. The lowest testing error recorded was 0.0541 . Therefore, it can be said that, based on the testing result, the ANFIS with three MF's had a better classification results compared to other ANFIS model that used "gbellmf".

Table 2. Comparison Of Error Rate For 2 MFs' AND 3 MF's With 2 Types Of MF's

\begin{tabular}{ccccc} 
Parameter of MF's & \multicolumn{2}{c}{ Number of MF's } & \multicolumn{2}{c}{ 3MF's } \\
& Training error (RMSE) & Testing error (RMSE) & Training error (RMSE) & Testing error (RMSE) \\
\hline Gauss & $\mathbf{0 . 2 9 5 6}$ & 1.6235 & 0.3902 & $\mathbf{0 . 0 5 4 1}$ \\
Gbell & 0.3237 & 1.1106 & 1.3156 & 0.3383 \\
\hline
\end{tabular}

(i) F1, F2, F3 MF's Before

nitial MFs on F1,F2 \& F3)

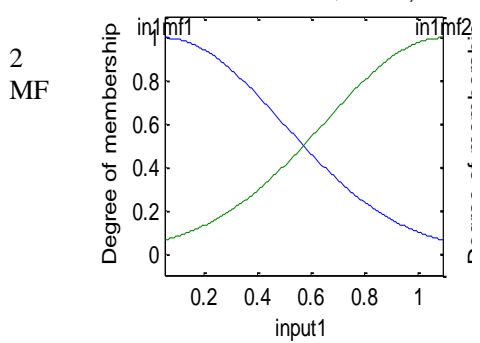

Initial MFs on F1,F2 \& F3

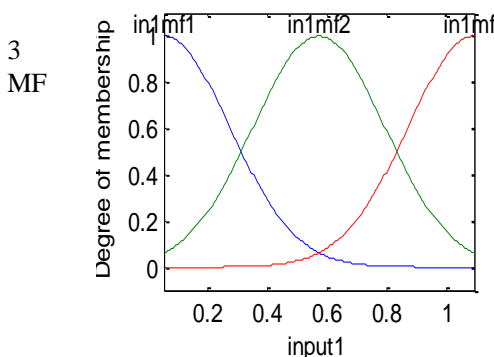

(i) F1, F2, F3 MF's Before
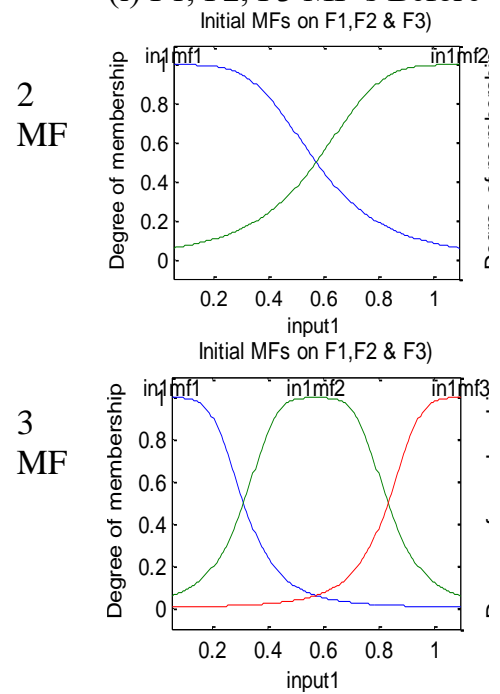

(ii) MF's on F1After

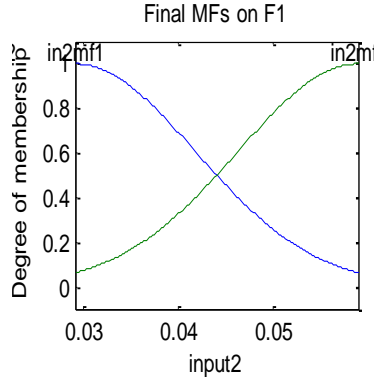

Final MFs on F1

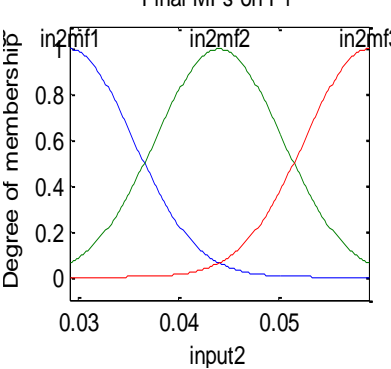

(iii) MF's on F2 After

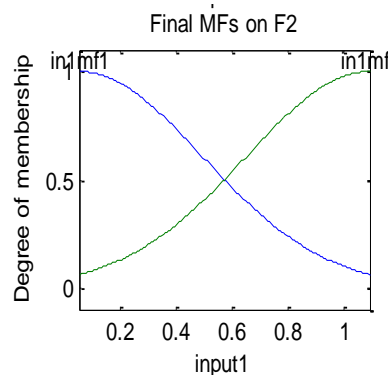

Final MFs on F2

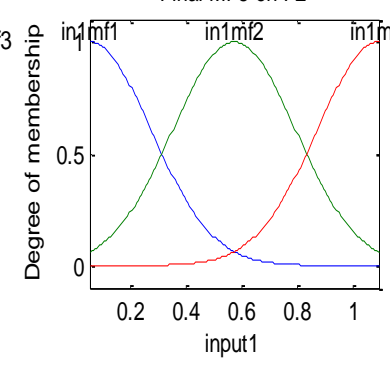

(a) "Gaussmf" (iv) MF's on F3 After

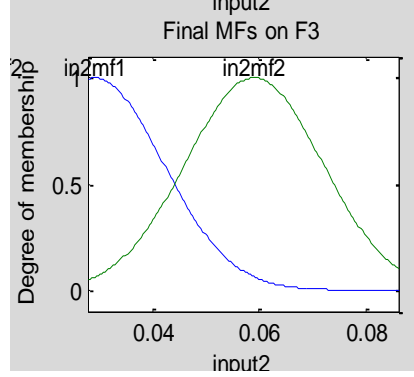

Final MFs on F3

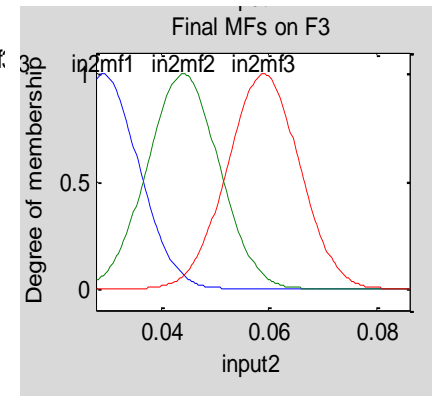

(ii) MF's on F1 After
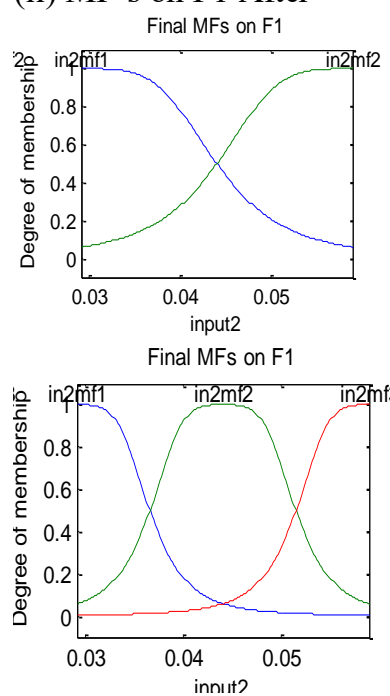

(iii) MF's on F2 After
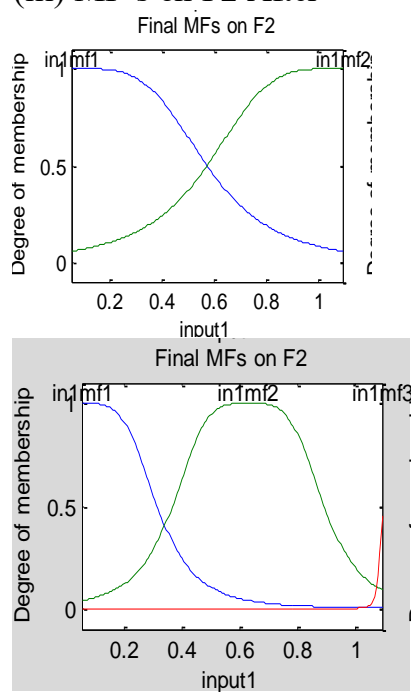

(iv) MF's on F3 After
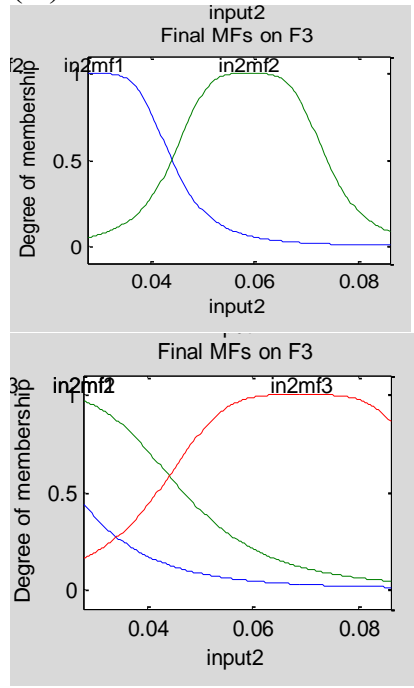

(b) "Gbellmf"

Figure 3. Plots for 2 MF's and 3 MF's (i) Initial MF's input and (ii-iv) Final MF's for all three inputs (F1, F2, F3) using two types of MF's (a) "Gaussmf”, and (b) “Gbellmf” 


\section{CONCLUSION}

In this work, it can be concluded that the analysis of fault classification disturbances based ANFIS classifier shows that "gaussmf" with three numbers of MF's has suitable method for fault disturbances classification. For this study, the use of three gaussian MFs ("gaussmf") has obtained the best classification results based on the lowest testing error rate. Therefore, this work shows that type of MF's and numbers of MF's play an important role in the analysis of the ANFIS classification. Furthermore, the ANFIS classifier can improve significantly by manipulating the type and number of the MFs.

\section{ACKNOWLEDGEMENTS}

The author would like to thank Faculty of Electrical Engineering and JPBSM UiTM for the support grant in publishing this manuscript.

\section{REFERENCES}

[1] M. Markovska and D. Taskovski, "Optimal Wavelet Based Feature Extraction and Classification of Power Quality Disturbances Using Random Forest,” no. July, pp. 6-8, 2017.

[2] S. Shahbudin, Z. F. Mohmad, S. I. Suliman, M. Kassim, and R. Mohamad, "Classification of power quality disturbances at transmission system using support vector machines," Indones. J. Electr. Eng. Comput. Sci., vol. 6, no. 2, pp. 310-317, 2017.

[3] K. Thirumala, A. C. Umarikar, and T. Jain, “A generalized empirical wavelet transform for classification of power quality disturbances,” 2016 IEEE Int. Conf. Power Syst. Technol., pp. 1-5, 2016.

[4] E. G. Ribeiro, G. L. Dias, B. H. G. Barbosa, and D. D. Ferreira, "Real-time system for automatic classification of power quality disturbances," 2016 17th Int. Conf. Harmon. Qual. Power, pp. 908-913, 2016.

[5] J. A. Bosnic, G. Petrovic, A. Putnik, and P. Mostarac, "Power quality disturbance classification based on wavelet transform and support vector machine," 2017 11th Int. Conf. Meas., pp. 9-13, 2017.

[6] X. Shicheng, X. Li, and B. Leping, "An effective S-Transform feature extraction method for classification of power quality disturbance Signals,” Proc. - 2015 Chinese Autom. Congr. CAC 2015, pp. 1555-1560, 2016.

[7] F. Ucar, O. F. Alcin, B. Dandil, and F. Ata, "Power quality event detection using a fast extreme learning machine," Energies, vol. 11, no. 1, pp. 1-14, 2018.

[8] S. M. B. a. S. P. L. C. Saikia, "Detection and Classification of Power Quality Disturbances Using Wavelet Transform, Fuzzy Logic and Neural Network," India Conference (INDICON), 2010 Annual IEEE, pp. 1 - 5, 2010.

[9] Mohamed M Ismail and M A. Moustafa Hassan. (2013). "Distance Relay Protection for Short and Long Transmission line." U2013 Proceedings of International Conference on Modelling, Identification \& Control (ICMIC): 204-211.

[10] T. S. Kamel, M. A. Moustafa Hassan, A. El-Morshedy (2009). "Advanced Distance Protection Scheme for Long Transmission Lines In Electric Power Systems Using Multiple Classified ANFIS Networks." IEEE Conference Publications: 1-5

[11] Adnan H. Tawafan, Marizan bin Sulaiman, Zulkiflie bin Ibrahim (2012). "Adaptive Neural Subtractive Clustering Fuzzy Inference System for the Detection of High Impedance Fault on Distribution Power System." International Journal of Artificial Intelligence 1(2): 63-72.

[12] H. a. F. Rasli, "Fault Diagnosis in Power Distribution Network Using Adaptive Neuro-Fuzzy Inference System (ANFIS)," Fuzzy Inference System - Theory and Applications, 2012.

[13] J.-S. R. Jang, "ANFIS: Adaptive Network Based Fuzzy Inference System," IEEE Transaction on Systems, Man and Cybernetic, vol. 23, pp. 665-685, 1993.

[14] J. S. R. Jang. C. T. Sun, E. Mizutani "A Computational Approach to Learning And Machine " Intelligence NeuroFuzzy and Soft Computing, Prentice Hall, US Edition, 1997

[15] Alex Wenda, A. Hussain, S. A. Samad, R. Mohamed abd M A Hannan "Web Based On Mobile Phone for Automatic Classification of Power Quality Disturbance Using the S-Transform and Support Vector Machine," Proceeding of International Technical Conference of IEEE Region 10 (TENCON 2010), pp. 21-24, 2010.

[16] Resul COTELI, "A Combined Protective Scheme for Fault Classification and Identification of Faulty Section in Series Compensated Transmission Lines," Turkish Journal of Electrical Engineering \& Computer Sciences, pp. 1-15,2013

[17] "'The Fuzzy Logic Toolbox' and 'Wavelet Toolbox' Matlab 7.0 " The Math Works.

[18] D. K. Mohanta, Sadhu, P.K, and Chakrabarti, R., "Fuzzy Markov Model for Determination of Fuzzy State Probabilities of Generating Units Including the Effect of Maintenance Scheduling," IEEE Trans Power Deliv, vol. 20, pp. 2117-2124, 2005.

[19] M. J. R. a. D. K. Mohanta, "Adaptive Neuro-Fuzzy Inference System Approach for Transmission Line Fault Classification and Location Incorporating Effects of Power Swings," The Institution of Engineering and Technology, vol. 2, pp. 235-244, March 2008.

[20] Tamer S. Kamel, M. A. Moustaffa Hassan, "Adaptive Neuro-Fuzzy Inference System (ANFIS) For Fault Classification in the Transmission Lines," The Online Journal on Electronics and Electrical Engineering (OJEEE), Vol. (2) - No. (1),164-169,2009 
[21] A. H. Shahrani Shahbudin, Ahmed Al-Shafie, N. M. Tahir, S. A. Samad, "Adaptive Neuro-Fuzzy Inference System For Human Posture Classification Using A Simplified Shock Graph," Visual Informatics: Bridging Research and Practice, pp. 585-595, 2009.

[22] E. D. Ubeyli "Adaptive Neuro-Fuzzy Inference Systems for Automatic Detection of Breast Cancer," Journal of Medical System, vol 33, pp. 353-358., 2009.

\section{BIOGRAPHIES OF AUTHORS}

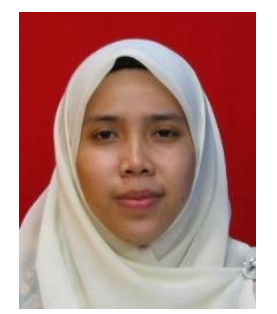

Shahrani Shahbudin received B.Eng.(Hons) degree from the University of Malaya, Kuala Lumpur in 1999. Master and Phd in 2004 and 2014 from Universiti Kebangsaan Malaysia respectively. She is currently working as a senior lecturer at the Centre for Computer Engineering Studies, Faculty of Electrical Engineering, University Teknologi MARA (UiTM) Shah Alam and member of MBOT, IEEE, IEM and BEM organization. Her main research interests include machine learning, deep learning, intelligent system, pattern classification and IoT for classification and visualization.

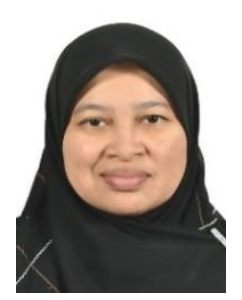

Murizah Kassim is currently working as senior lecturer at Faculty of Electrical Engineering, Universiti Teknologi MARA, Shah Alam, Selangor. She received her PhD in Electronic, Electrical and System Engineering in 2016 from the Faculty of Built Environment and Engineering, Universiti Kebangsaan Malaysia (UKM), Malaysia. She has published many papers related to computer network, Internet of Things (IoT), Web and mobile development applications research. She has experienced about 19 years in technical team at the Centre for Integrated Information System, UiTM Shah Alam. She is also the member of Enabling Internet of Things Technologies (ElIoTT) research group UiTM. She joined the academic since January 2009 and currently member of IEEE, IET, IAENG and IACSIT organization.

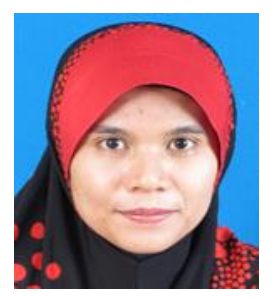

R. Mohamad obtained the B. Eng. degree in Electrical Engineering and M. Eng. Science degree from Universiti Malaya, Kuala Lumpur in 2003 and 2008 respectively. She received the PhD. degree in Aerospace Engineering from Universiti Putra Malaysia, in 2016. From 2006 until now, she is with Faculty of Electrical Engineering, Universiti Teknologi MARA, as a senior lecturer. She has been appointed as a Head of Computer Engineering Studies at the same institution since 2018. Her research interests are in computing algorithm and digital signal processing for deep space communication, channel coding, theory of computation, and wireless communication.

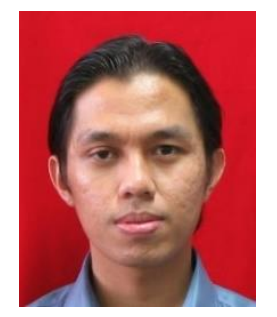

Saiful I Suliman is a senior lecturer at the Faculty of Electrical Engineering, Universiti Teknologi MARA (UiTM), Malaysia. He obtained $1^{\text {st }}$ class degree in Artificial Intelligence in 2002 and Master of Science (Electrical Engineering) from UiTM in 2006. His Phd was awarded by The University of Nottingham, UK in 2015. His research interest is mainly in the area or artificial intelligence, optimization and pattern recognition algorithm, metaheuristic approach, frequency bandwidth spectrum and power system operations. He has published papers in many international conferences as well as reputable journals. $\mathrm{He}$ is a certified Professional Technologist and Chartered Engineer (CEng) with IET, UK. He is currently the head of ICT Unit, Institute of Research Management and Innovation at UiTM.

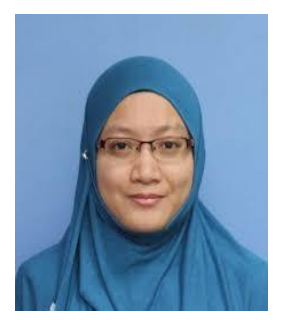

Y.W. Mohamad Yusof obtained her B. Eng (Hons) degree in Electronics Engineering from Universiti Teknologi MARA in 2004 and Master Science degree from Universiti Sains Malaysia in 2006. From 2006 until now, she is with Faculty of Electrical Engineering, Universiti Teknologi MARA, as a senior lecturer. Her research interests are in IOT applications, digital image and signal processing. 